\title{
1 The evolution of realized niches within freshwater Synechococcus
}

2

3 lineage of Cyanobacteria.

3- mathieu.castelli@gmail.com nicolas.tromas@umontreal.ca

Authors: Nicolas Tromas ${ }^{1 \# *}$, Zofia E. Taranu ${ }^{2 \#,}$ Mathieu Castelli3" ${ }^{\sharp}$, Juliana S. M. Pimentel ${ }^{4}$, Daniel A. Pereira ${ }^{4}$, Romane Marcoz ${ }^{1}$, B. Jesse Shapiro ${ }^{1 *}$ and Alessandra Giani ${ }^{4}$

1- Département de sciences biologiques, Université de Montréal, 90 Vincent-d'Indy, Montréal, QC, Canada, Montréal, QC H2V 2S9, Canada

2- Environnement et Changement Climatique Canada,105 Rue McGill, Montréal, QC H2Y 2E7

4- Federal University of Minas Gerais, Belo Horizonte, Minas Gerais, Brazil

*Corresponding authors: B. Jesse Shapiro. Phone: 514-343-6033. E-mail: jesse.shapiro@umontreal.ca; Nicolas Tromas. Phone 514-343-3188. E-mail:

\# These authors contributed equally to this work

\section{Originality-Significance Statement}

We address a fundamental question in ecology and evolution: how do niche preferences change over evolutionary time? Using time-series analysis of $16 \mathrm{~S}$ rRNA gene amplicon sequencing data, we develop an approach to highlight the importance of biotic factors in defining realized niches, and show how niche preferences change proportionally with the $16 \mathrm{~S}$ gene molecular clock within the genus Synechococcus. Ours is also one of few studies on the ecology of freshwater Synechococcus, adding significantly to our knowledge about this abundant and widespread 


\section{Summary}

34 Understanding how ecological traits have changed over evolutionary time is a fundamental

35 question in biology. Specifically, the extent to which more closely-related organisms share

36 similar ecological preferences due to phylogenetic conservation - or if they are forced apart by

37 competition - is still debated. Here we explored the co-occurrence patterns of freshwater

38 cyanobacteria at the sub-genus level to investigate whether more closely-related taxa share more

39 similar niches, and to what extent these niches were defined by abiotic or biotic variables. We

40 used deep 16S rRNA gene amplicon sequencing and measured several abiotic environmental

41 parameters (nutrients, temperature, etc.) in water samples collected over time and space in Furnas

42 Reservoir, Brazil. We found that relatively more closely-related Synechococcus (in the

43 continuous range of $93-100 \%$ nucleotide identity in $16 \mathrm{~S}$ ) had an increased tendency to co-occur

44 with one another (i.e. had similar realized niches). This tendency could not be easily explained by

45 shared preferences for measured abiotic niche dimensions. Thus, commonly measured abiotic

46 parameters might not be sufficient to characterize, nor to predict community assembly or

47 dynamics. Rather, co-occurrence between Synechococcus and the surrounding community

48 (whether or not they represent true biological interactions) may be a more sensitive measure of

49 realized niches. Overall, our results suggest that realized niches are phylogenetically conserved,

50 at least at the sub-genus level and at the resolution of the $16 \mathrm{~S}$ marker. Determining how these

51 results generalize to other genera and at finer genetic resolution merits further investigation. 
58 A bacterial community is a group of potentially interacting organisms that coexist at a particular

59 place and time (Magurran, 2003). Environmental selective pressures are a strong force shaping

60 microbial community assembly (Martiny et al, 2015). We know, for example, that certain abiotic

61 factors explain a large portion of the variation in microbial community composition (e.g. the

62 effect of $\mathrm{pH}$ on soil bacterial communities; Fierer and Jackson, 2006). Therefore, associations

63 between microbial traits - generally defined as a phenotypic response to a specific environmental

64 condition - and abiotic niches could help explain community assembly rules (Green et al., 2008;

65 Burke et al., 2011). However, using trait-based approaches to understand communities may be

66 challenging, as important abiotic variables may go unmeasured. Even less is known about biotic

67 interactions, despite their importance in determining community composition, diversity, and

68 dynamics (Needham et al., 2016).

70 Abiotic factors are generally thought to determine an organism's fundamental niche (where it is

71 theoretically capable of living), whereas biotic factors determine its realized niche (where it

72 actually lives in nature; Hutchinson, 1957). Species (or taxonomic) co-occurrence networks are

73 often used to infer niche similarity among organisms that tend to co-occur in nature over space

74 and time, and microbial co-occurrence networks can easily be constructed from 16S rRNA gene

75 amplicon sequencing surveys (Friedman and Alm, 2012; Röttjers and Faust, 2018; Tromas et al.,

76 2018). However, co-occurrence can be driven by both abiotic and biotic factors, which are hard

77 to disentangle in practice (Kraft et al., 2015). Regardless, organisms sharing a similar niche are

78 expected to be associated with similar surrounding communities (Cohan and Koeppel, 2008;

79 Faust et al.2012; Pascual-García et al., 2014). 
81 A fundamental question spanning ecology and evolution is how ecological traits change over

82 evolutionary time. For example, some traits (such as bacteriophage host range or phosphorus

83 utilization) evolve rapidly at the tips of a phylogenetic tree, whereas other traits (such as salinity

84 preference) are deeply conserved (Martiny et al. 2015). When more closely related organisms

85 share similar ecological preferences, so-called "habitat filtering" or "environmental" filtering is

86 expected to result in phylogenetic clustering, meaning that a community tends to contain more

87 closely related organisms than expected by a random draw from the phylogeny (Webb et al.,

88 2002; Horner-Devine \& Bohannan, 2006; Martiny et al., 2015). In contrast, if close relatives

89 evolve different traits to avoid competitive exclusion, this will result in phylogenetic

90 overdispersion (i.e. a community composed of more distant relatives than expected by chance).

91 However, the relative importance of these two processes in shaping microbial communities is still

92 widely debated, and difficult to distinguish (Koeppel and Wu, 2014; Cadotte and Tucker, 2017).

93 We have previously shown that within the cyanobacterial genus Dolichospermum (Tromas et al.,

94 2018), the relationships between phylogenetic distance and ecological similarity varies by trait,

95 suggesting that it might be necessary to analyze each niche dimension or trait separately (Martiny

96 et al., 2015).

98 In this study, we explored the co-occurrence patterns of freshwater cyanobacteria at the sub-

99 genus level, to investigate if more closely related taxa have more similar niches, and to what

100 extent these niches can be quantified by abiotic or biotic variables. We use the term "closely

101 related" as a relative term, describing a continuous range of genetic similarity (93-100\%

102 nucleotide identity) in 16S rRNA gene sequences. We focused on Synechococcus, the most

103 abundant cyanobacterial genus in Furnas Reservoir (Brazil) at the time of sampling (2006-2008). 
104 Synechococcus is among the most abundant organisms living in oceans and lakes (Stockner et al.

105 2000; Scanlan, 2003). The phylogenetic coherence of the genus has been questioned by a recent

106 study showing it to be polyphyletic (Coutinho et al 2016). Synechococcus is physiologically

107 highly plastic, ubiquitous, and able to acclimate to different environmental conditions (Callieri

108 1996; Vörös et al. 1998; Callieri et al., 2011). Previous studies have shown that different

109 Synechococcus strains could co-exist in the same site but respond differently to environmental

110 changes, suggesting niche partitioning (Ferris et al., 2003; Allewalt et al., 2006; Becker et al.,

111 2007; Becraft et al., 2011; Callieri et al. 2012). Recently, Zheng et al. (2018), observed a

112 geographical pattern of heterotrophic bacteria associated with different marine Synechococcus

113 strains, indicating that strains living in the same area tend to be associated with similar

114 communities. It remains, however, unclear whether the Synechococcus genotype or the

115 environment are the main drivers of Synechococcus interactions with the surrounding microbial

116 community.

118 Using deep 16S rRNA gene amplicon sequencing of 86 water samples collected in time series

119 across nine locations in the Furnas Reservoir, we tracked genetic diversity within the

120 Synechococcus genus, along with the surrounding microbial community, and measured several

121 abiotic variables. We found that that more closely-related Synechococcus tended to co-occur with

122 one another and also with similar surrounding microbial communities. Such phylogenetic

123 clustering indicates that realized niche similarity tends to evolve proportionally with substitutions

124 in the $16 \mathrm{~S}$ gene in the Synechococcus lineage (i.e. niches evolve according to the $16 \mathrm{~S}$ gene

125 molecular clock). However, more closely-related Synechococcus did not have similar abiotic

126 niche preferences (with the exception of total phosphorus). These results suggest that biotic

127 factors may be strong niche determinants, which could be particularly useful when relevant 
128 abiotic factors are unknown or cannot be readily measured. Alternatively, cryptic abiotic drivers

129 may determine niche and community structure, but biotic factors provide the most informative

130 measure of niche similarity.

\section{Materials and methods}

\section{Sampling, environmental data measurements}

135 A total of 90 water samples were collected from September 2006 to April 2008 at nine stations in

136 Furnas Reservoir (Minas Gerais, Brazil). Furnas is a large reservoir $\left(1440 \mathrm{~km}^{2}\right)$, located in

137 southeastern Brazil $\left(20^{\circ} 40^{\prime} \mathrm{S} ; 46^{\circ} 19^{\prime} \mathrm{W}\right)$ and formed by the damming of two main rivers (Rio

138 Grande and Rio Sapucaí), which divide the reservoir in two separated branches (Figure S1).

139 Sampling stations F1, F4, F6, F9 are from a relatively pristine branch of the reservoir, whereas

140 F12, F14, F18 and F20 are impacted by human activities. Temperature profiles were measured in

141 the water column by aid of a multi-parameter probe (556 YSI, USA). Water samples were

142 collected from the euphotic zone (determined by Secchi disc depth) by a Van Dorn vertical water

143 sampler. Samples were stored in bottles that had been acid-washed and rinsed with deionized

144 water. A portion of each water sample was immediately filtered through glass fiber filters

145 (Whatmann GF/F, $0.7 \mu \mathrm{m}$ pore size). The exact filtered volumes were recorded and filters were

146 kept frozen until further analyses (chlorophyll a, DNA and microcystin). For dissolved nutrient

147 analyses, $200 \mathrm{~mL}$ of filtered water samples were stored at $-20{ }^{\circ} \mathrm{C}$. For total phosphorus, samples

148 were frozen with no previous filtration. Nutrient analyses were performed using

149 spectrophotometric methods according to APHA (2005). All nutrient analyses (Nitrate, Nitrite

150 and total phosphorus (TP) were performed on three replicates. 
153 DNA was extracted from frozen filters according to Kurmayer et al. (2003), with few

154 modifications. Briefly, filters were treated with a sucrose buffer $(25 \% \mathrm{w} / \mathrm{v}$ sucrose, $50 \mathrm{mM}$ Tris-

$155 \mathrm{HCl}, 100 \mathrm{Mm}$ EDTA, $\mathrm{pH} 8)$ on ice for $2 \mathrm{~h}$ and with addition of lysozyme $\left(5 \mathrm{mg} / \mathrm{mL}, 1 \mathrm{~h}, 37^{\circ} \mathrm{C}\right)$.

156 Proteinase $\mathrm{K}(100 \mu \mathrm{g} / \mathrm{mL})$ in sodium dodecyl sulfate $(2 \% \mathrm{v} / \mathrm{v})$ was added and filters were

157 incubated overnight at $55^{\circ} \mathrm{C}$. A phenol:chloroform:isoamyl alcohol solution $(25: 24: 1$, v/v) was

158 used for protein precipitation and DNA isolation. The DNA was cleaned in 100\% ethanol and

159 pellets rinsed with $70 \%$ ethanol. The DNA was resuspended in TE $(10 \mathrm{mM}$ Tris- $\mathrm{HCl}, \mathrm{pH}$, and

$1601 \mathrm{mM}$ EDTA). The DNA extract was quantified by a spectrophotometer (Perkin Elmer, Lambda

$16125)$, at $260 \mathrm{~nm}$ and $280 \mathrm{~nm}$, and its quality checked in 1\% (w/v) agarose gel, stained with

162 ethidium bromide. DNA samples were stored at $-20^{\circ} \mathrm{C}$.

\section{Sequence analysis}

165 We followed the same protocol described in Tromas et al., (2017) to generate a library of

166 V4 region amplicons. Libraries for 2x250bp paired-end Illumina sequencing were prepared using

167 a two-step 16S rRNA gene amplicon PCR as described in Preheim et al. (2013). We performed

168 one sequencing run using MiSeq reagent Kit V2 (Illumina, San Diego, CA, USA) on a MiSeq

169 instrument (Illumina). A total of 4,476,747 sequences of the $16 \mathrm{~S}$ rRNA gene V4 region were

170 obtained from 90 lake samples, two negative controls, and two mock community samples. We

171 obtained a median of 37,682 sequences per sample. Using a similar approach as described in

172 Tromas et al., (2018), we processed the sequences with SmileTrain

173 (https://github.com/almlab/SmileTrain/wiki; Preheim et al., 2013) for read quality filtering,

174 primer removal, chimera filtering, and merging using USEARCH (version 7.0.1090,

175 http://www.drive5.com/usearch/, default parameter) (Edgar, 2010), Mothur (version 1.33.3)

176 (Schloss et al., 2009), and Biopython (version 2.7). Minimum Entropy Decomposition (MED) 
177 was then applied to the filtered and merged reads to partition sequence reads into MED nodes

178 (Eren et al., 2015). MED was performed using the following parameters: -M noise filter set to

1791000 , resulting in $\sim 17.5 \%$ of reads filtered and 466 MED nodes representing the whole bacterial

180 community. Samples with less than 1000 reads were removed, yielding a final dataset of 86

181 reservoir samples. Finally, we assigned taxonomy to MED nodes using the assign_taxonomy.py

182 QIIME script (default parameters), and a combination of GreenGenes and a freshwater-specific

183 database (Freshwater database 2016 August 18 release; Newton et al., 2011), using TaxAss

184 (https://github.com/McMahonLab/TaxAss, installation date: September 13 ${ }^{\text {th }}$ 2016; Rohwer et al.,

185 2017). We found a total of 40 Synechococcus nodes, which we refer to as strains below.

187 Spatio-temporal analysis

We used multivariate regression tree analyses (Breiman et al. 1984; De'ath 2002) to

189 investigate if spatio-temporal variables could explain genetic variation within Synechococcus. We

190 used two different temporal predictors: year and month and one spatial predictor: station. The

191 analysis was performed using the function mvpart() and rpart.pca() from the $\mathrm{R}$ mvpart package

192 (Therneau and Atkinson, 1997; De'ath, 2007). Prior to analysis, the Synechococcus MED node

193 table was Hellinger transformed to downweight the effect of double-zeros (Rao, 1995).

Genetic distance between Synechococcus nodes

We measured the genetic distance between Synechococcus MED nodes using the software

197 MEGA (Kumar et al., 2016; version 7.0.18) with the p-distance (the proportion of nucleotide

198 sites at which two sequences differ), calculated by dividing the number of sites with nucleotide

199 differences by the total number of sites compared (excluding sites with gaps). 


\section{Co-response to abiotic factors}

As described in Tromas et al. (2018), we used a Latent Variable Model (LVM)

203 framework (boral package in R; Hui 2015, Warton et al. 2015) to explore how Synechococcus

204 nodes co-responded to abiotic gradients and used these co-responses as indicators of niche

205 similarity. That is, for each abiotic factor, we ran separate LVMs, regressing the bacterial

206 community as a function (both linear and non-linear) of the given factor. This component of the

207 LVM thus defined the taxon-specific environmental responses. To then identify remaining

208 patterns of co-occurrence after accounting for all measured environmental variables, we fit a

209 global LVM which included all abiotic factors and two latent variables (sensu Letten et al. 2015

210 and Warton et al. 2015). To visualize patterns of co-occurrence arising from the different

211 environmental factors, we calculated two types of correlation matrices. The first, a co-response

212 correlation matrix, was constructed by calculating, for any two nodes, the correlation between

213 their fitted values. This correlation matrix thus represented the correlation between nodes that can

214 be attributed to a shared or diverging environmental responses. A significant positive correlation

215 between the fitted response of any two taxa to an environmental variable represented a co-

216 response (i.e., as one taxa increases in response to an environmental variable, the other likewise

217 increases), whereas a significant negative correlations between the fitted response of two taxa

218 represented some degree of niche separation (i.e., as one taxa increases in response to an

219 environmental variable, the other decreases).

221 To account for the correlation between nodes that may be attributable to biotic processes or

222 missing environmental covariates, a second type of correlation matrix, a residual correlation

223 matrix, was calculated using the latent variable coefficients of the global LVM. Since the latent

224 variables are the output of an ordination of the residuals, we expected weak residual correlations 
225 induced by the latent variables if the environmental variables were the dominant force structuring

226 patterns of species co-occurrence (i.e. the model explained most of the variance, with little left

227 unexplained in the residuals). Conversely, if any unmeasured environmental factors and/or biotic

228 processes are equally, or more, important than measured environmental factors, we expected

229 strong correlations based on the latent variables (i.e. the model was poor and much of the

230 observed variability remained in the residuals).

231 To test how Synechococcus niche similarity varied with genetic distance, we examined the

232 relationship between the co-response of Synechococcus taxa to environmental parameters and

233 their genetic distance (i.e., plotting the correlation coefficient of the LVM co-responses vs.

234 genetic distances) (R_script1). However, given the large number of environmental factors driving

235 phytoplankton community dynamics (Hutchinson, 1961), we expected some degree of co-

236 limitation whereby the response of a taxon to an environmental variable (and consequently the

237 degree of co-response among any two taxa) would be limited by other environmental variables.

238 In such cases, the taxon's response and rate of change would have an upper limit (set by all

239 measured environmental factors) but may not reach this limit if other, unmeasured factors are co-

240 limiting (Cade and Noon 2003). As an increasing number of unmeasured factors become limiting

241 at some sample location, or time point, the relationship between the response and the measured

242 factor becomes increasingly heterogeneous or wedge shaped. When such heterogeneous

243 variances are observed (wedge shape biplot), it suggests that there is not a single slope coefficient

244 that characterizes the relationship, and that focusing solely the coefficient fit by ordinary least

245 squares regression (mean response) may underestimate the true rate of change. Thus, to

246 accurately examine co-limitations among measured and unmeasured factors, we applied quantile

247 regressions using 'quantreg' $\mathrm{R}$ package (Koenker, 2015) (R_script1), which, instead of fitting a

248 regression to the mean response (ordinary least squares regression), fits regression curves to other 
249 quantiles of the response variable (Cade and Noon 2003). Finally, to provide an effect size for the

250 linear quantile relationships of abiotic co-responses vs. genetic distance, we calculated the

251 goodness of fit measure for quantile regression for a suite of quantiles (where the goodness of fit

252 is estimated as 1 minus the ratio between the sum of absolute deviations of the parameterized

253 model and the sum of absolute deviations of the unparameterized, null model). Note that this

254 goodness of fit measure (referred hereafter as R1) is not comparable to the OLS coefficient of

255 determination (R2); it is based on the variance of absolute deviations as opposed to the variance

256 of squared deviations, and consequently, R1 is always smaller than R2 (Koenker and Machado,

257 1999).

258

259

Co-occurrence with biotic factors

A caveat of the above LVM-quantile regressions is that although it identifies whether

261 some relationships are limited by unmeasured abiotic and/or biotic factors, it could not tease apart

262 the relative importance of each type of factor (everything is instead lumped as an unknown). In

263 order to determine the relative contribution of biotic processes, we thus quantified node co-

264 occurrence patterns among Synechococcus and the remaining community. In particular, we

265 explored the relationship between Synechococcus nodes and the surrounding community by

266 measuring: (1) co-occurrences between Synechococcus and other taxa, and (2) paired differences

267 in co-occurrence among Synechococcus nodes and a specific taxon. For the former, we calculated

268 co-occurrences among taxa using SparCC (Friedman and Alm, 2012), including 20 iterations to

269 estimate the median correlation of each pair of MED nodes, 500 bootstraps to assess the

270 statistical significance and centered log ratio (CLR) transform to correct for compositionality.

271 Correlations were then filtered using a false discovery rate threshold $(Q<0.05)$. For the latter, we

272 further calculated the absolute difference of SparCC correlation $(r)$ between a Synechococcus 
273 node $\left(\mathrm{X}_{\mathrm{i}}\right)$ and a specific taxon $\mathrm{T}$, such that $|\Delta r|=\left|\operatorname{Corr}\left(\mathrm{X}_{1}, \mathrm{~T}\right)-\operatorname{Corr}\left(\mathrm{X}_{2}, \mathrm{~T}\right)\right|$ where $\operatorname{Corr}$ is

274 defined here as the SparCC correlation between $\mathrm{X}_{\mathrm{i}}$ and $\mathrm{T}$.

275 For each non-Synechococcus taxon, we then estimated the relationship between $|\Delta r|$ and

276 the distance between the given Synechococcus nodes $\left(\mathrm{X}_{1}\right.$ and $\left.\mathrm{X}_{2}\right)$. A positive correlation would

277 indicate that more closely related Synechococcus nodes have a lower $|\Delta r|$ than more distant

278 nodes, i.e. more closely related nodes would have more similar correlations with potentially

279 interacting community members. To reduce potential bias or noise due to a small sample size of

280 Synechococcus pairs, we selected all non-Synechococcus nodes that were significantly co-

281 occurrent with at least seven different Synechococcus. This cutoff was chosen to ensure at least

28220 unique pairs of Synechococcus for each non-Synechococcus taxon. A total of 373 non-

283 Synechococcus taxa were selected using this cutoff, and the correlation between $|\Delta r|$ and genetic

284 distance was non-significant (Spearman correlation, $\mathrm{P}<0.05$ ) for 120 of them. Finally, we

285 performed a permutation test to quantify any bias in the method due to data structure. For each of

286 the significant non-Synechococcus taxa, we estimated the rate of false positive correlations by

287 sampling values of Synechococcus genetic distance with replacement, while randomizing the

288 association between genetic distance and $|\Delta r|$. We performed 1000 permutations for each

289 significant non-Synechococcus taxon using the script R_script2. We then recorded the proportion

290 ( $p$ ) of permutations yielding a larger correlation than observed, after adding a pseudocount of 1 to

291 both the numerator (the number of permutations yielding a larger correlation than observed) and

292 the denominator (the total number of permutations).

293

294

295

296

\section{Phylogenetic analysis}

We first verified that our Synechococcus sequences were monophyletic by building a

297 phylogenetic tree with FastTree (version 2.1.8, Price et al., 2009), using all MED node sequences 
298 aligned with MAFFT (v7.154b; Katoh and Standley, 2013). We then specifically aligned

299 Synechococcus MED node sequences using muscle (in Mega, version 7.0.26; Kumar et al., 2016)

300 and built phylogenetic trees using PhyML with the general time-reversible (GTR) model of

301 nucleotide substitution (version 3.0; Guindon et al., 2010). We used the ALDEx2 R package

302 (version: 1.5.0; Fernandes et al., 2014) and the aldex() function to identify MED nodes associated

303 with the pristine or human-impacted branches of Furnas Reservoir. To do so, we used Welsh's t-

304 test and 128 Monte Carlo samples. ALDEx2 uses the CLR transformation to avoid compositional

305 effects. A Q-value below 0.05 after Benjamini-Hochberg correction was taken as evidence for

306 association. We also used a second association method, DESeq2 implemented in the

307 “MicrobiomeAnalyst” web-based tool (Dhariwal et al., 2017).

308

309

Statistical analysis

310

311 All statistical tests were performed in R.

312 


\section{Results}

Abiotic factors shape Synechococcus community structure

317 Furnas Reservoir is divided into two branches: one, which is heavily human-impacted (Figure S1,

318 sampling stations 12-20) and the other, less impacted (stations 1-9). Synechococcus dominated

319 the bacterial community in both branches during the sampling period (2006-2008) (Figure S2)

320 and formed a monophyletic group (Figure S3, S4). Furnas Reservoir is meso-eutrophic based on

321 phosphorus concentrations. Total phosphorus (TP) did not vary significantly across stations but

322 did vary significantly within each station over time (Figure S5). Temperature did not vary widely

323 across sampling stations or time. We first asked whether particular Synechococcus strains (which

324 we used interchangeably with MED nodes) were associated with different branches of the

325 reservoir, different time periods, or different abiotic factors. We applied a multivariate regression

326 tree analysis (Breiman et al. 1984; De'ath 2002) to test whether the composition of

327 Synechococcus strains changed over time and space. We found that the year and month of

328 sampling did not explain much variance $\left(\mathrm{R}^{2}=0.02\right.$ and $\mathrm{R}^{2}=0.03$, respectively), nor did the station

$329\left(\mathrm{R}^{2}=0.06\right)$ or branch of the reservoir $\left(\mathrm{R}^{2}=0.02\right)$. In contrast, Nitrite, $\mathrm{TP}$, depth, and water

330 temperature all explained a significant proportion of the variance (Figure S6; 22.5\% of variance

331 explained). Nitrite is the single most explanatory variable (13\% of variance, but also colinear

332 with temperature and depth; Figure S6), followed by TP (5.5\% of variance; Figure S7).

334 More closely related Synechoccus do not share similar preferences for most measured abiotic

335 niches

336 Having established that abiotic factors, but not temporal or spatial variation, explain a significant

337 amount of variation in the relative abundances of Synechococcus strains, we asked whether more 
338 closely related strains tended to share similar abiotic niches. We examined Synechococcus 16S

339 sequences across a range of $\sim 93-100 \%$ nucleotide identity, which is a rather large range

340 considering that niche partitioning can occur at relatively high identity, $>97 \%$ (Martiny et al.

3412015 ; Larkin and Martiny, 2017). Here we defined abiotic niche similarity by estimating the co-

342 responses of pairs of Synechococcus strains to each abiotic factor using LVM-quantile regression

343 (Methods). For most abiotic factors, we did not observe any significant relationship between co-

344 responses and genetic distance, with the exception of total phosphorus for which the relationship

345 was negative (Median p-value across quantiles $=0.021$; median R1 across quantiles $=0.12$ )

346 (Figure $1 \&$ S8, Table S2). Similarly, the degree of abiotic niche separation (i.e. significant

347 negative correlations between the fitted response of any two Synechococcus strains to an abiotic

348 factor) was generally independent of genetic distance (Figure S9, S10), except for depth and

349 Nitrite which were significant at some quantiles but with quite a poor fit (median R1 across

350 quantiles $\leq 0.02)($ Table $\mathrm{S} 2 \mathrm{C})$.

351 We also evaluated non-linear quantile relationships among co-responses and genetic

352 distances, but we never found a significant non-linear relationship over the total range of genetic

353 distances (Table S2A-C). We repeated the quantile regression analysis in a restricted range of

$354 \quad 100-97 \%$ identity, to see if any linear trend is discernible in a range roughly corresponding with

355 species or genera (Figure S11). The negative linear trend observed between co-responses and

356 genetic distance for TP in the full range of genetic identify (Figure 1) was still observed in the

357 restricted range (Figure S11) although not significant at all quantiles (Table S2D). There was also

358 some evidence for a non-linear relationship between genetic distance and both positive and

359 negative co-responses to TP (Table S2 D, E), suggesting possible niche separation among closely

360 related ( $\sim 99 \%$ identical) strains. However, this trend is driven by just three pairs of very closely

361 related strains with quite different TP preferences (Figure S12). Overall, these results suggest that 
362 TP-related niches show some evidence for phylogenetic conservation within freshwater

363 Synechoccus, while the evidence for other niches is weak or inconclusive.
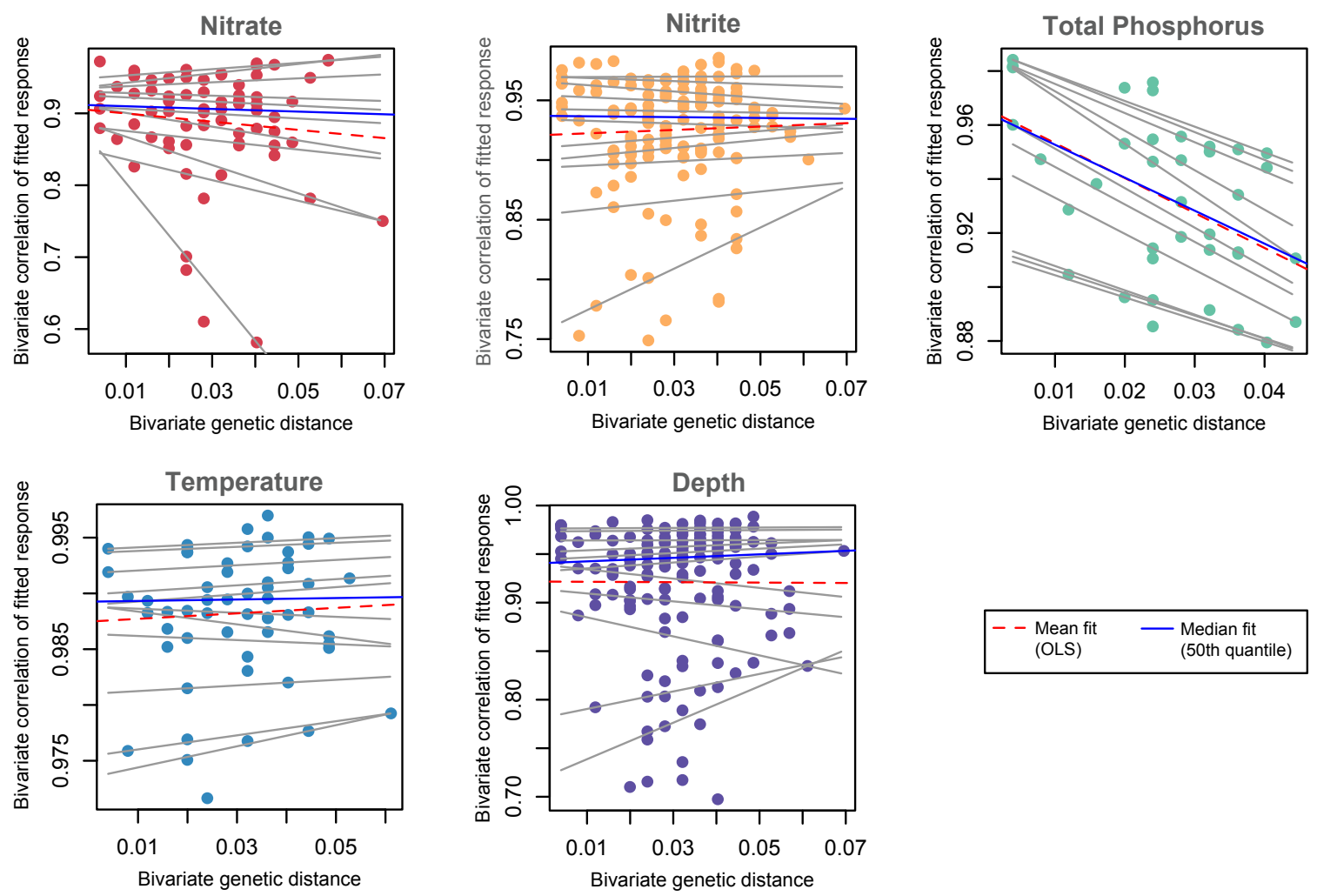

365 Figure 1. Relationship between Synechococcus niche similarity and genetic distance. Niche similarity is defined as a positive LVM co-response (y-axis). Shown are the scatterplots of LVM co-responses versus each variables superimposed with the lines for $5^{\text {th }}, 10^{\text {th }}, 15^{\text {th }}, 25^{\text {th }}, 35^{\text {th }}, 45^{\text {th }}$, $55^{\text {th }}, 65^{\text {th }}, 75^{\text {th }}, 85^{\text {th }}$ and $90^{\text {th }}$ quantile regression fits (grey lines, for each co-response quantile), the median fit $\left(50^{\text {th }}\right.$ quantile; blue line), and the least squares estimate or mean fit (dashed red 370 line). Biotic processes or unmeasured environmental variables could define Synechococcus realized

373 niches, and are captured in the residual variation of the global LVM. We next asked whether 374 more closely-related Synechococcus strains had more similar co-responses to this residual 375 variation. For the positive co-responses (Figure 2A, B), we observed a decrease with genetic 
376 distance. This result suggests a negative relationship between the residuals (i.e. unmeasured

377 abiotic variables and/or biotic niche preferences) and phylogenetic distance. For the negative

378 residual correlations (Figure 2C, D), there was no apparent relationship with genetic distance.

379 Therefore, there remain significant co-responses that were not explained by the abiotic factors

380 measured in this study. Overall, most of the measured abiotic environmental variables were not

381 related to Synechococcus genetic distance. However, preferences for unmeasured biotic or abiotic

382 factors do tend to change with genetic distance, such that more closely-related Synechococcus

383 have similar realized niche preferences. 
(a)

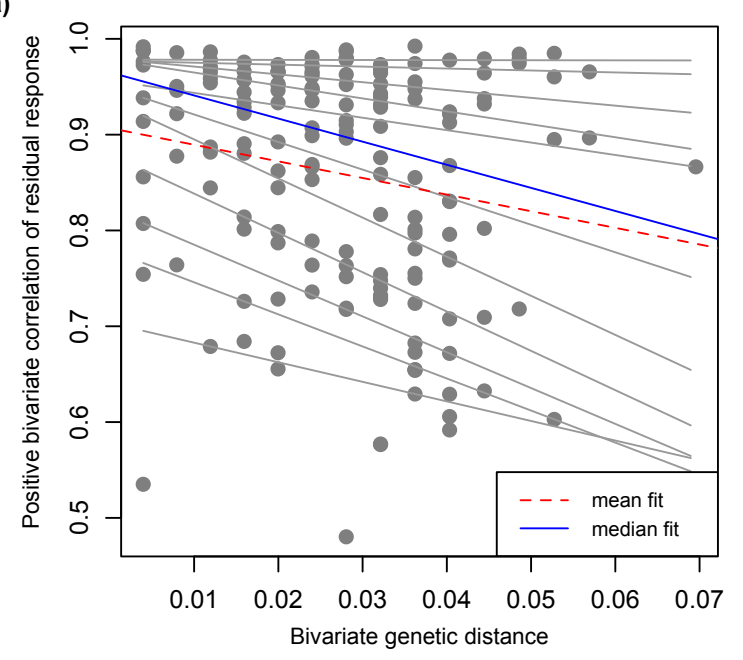

(b)

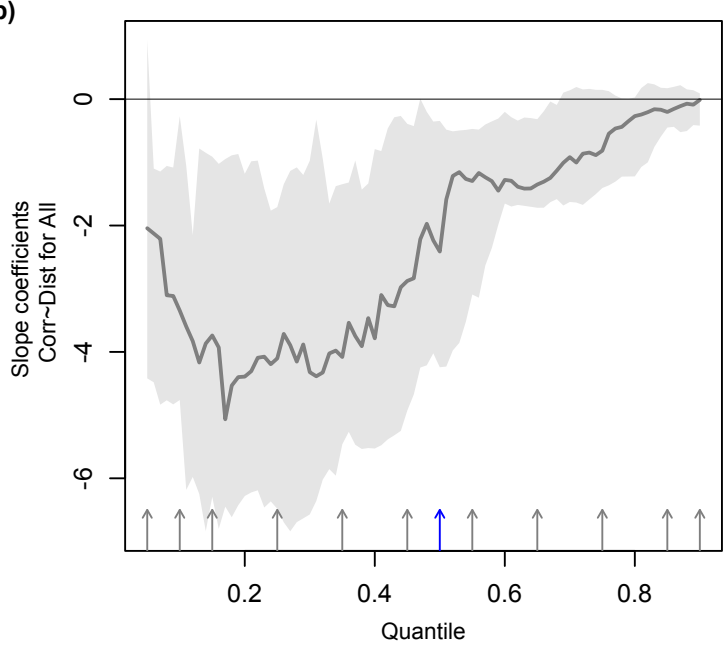

(c)

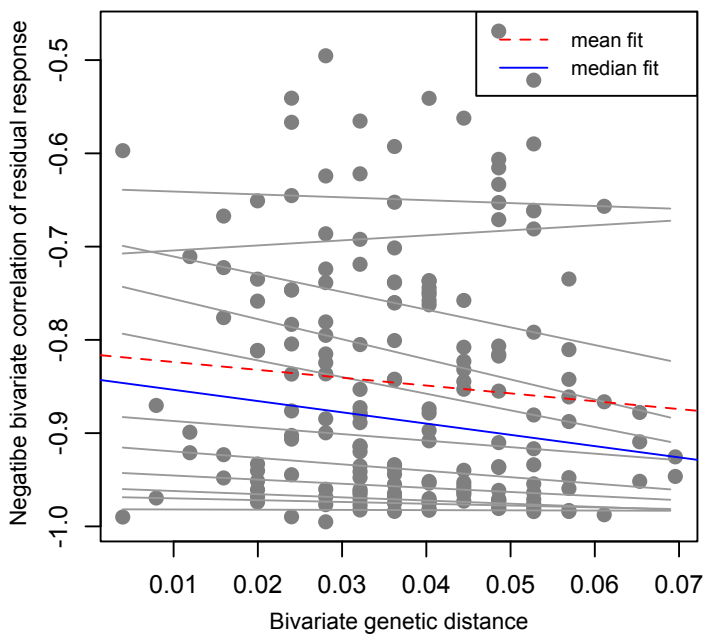

(d)

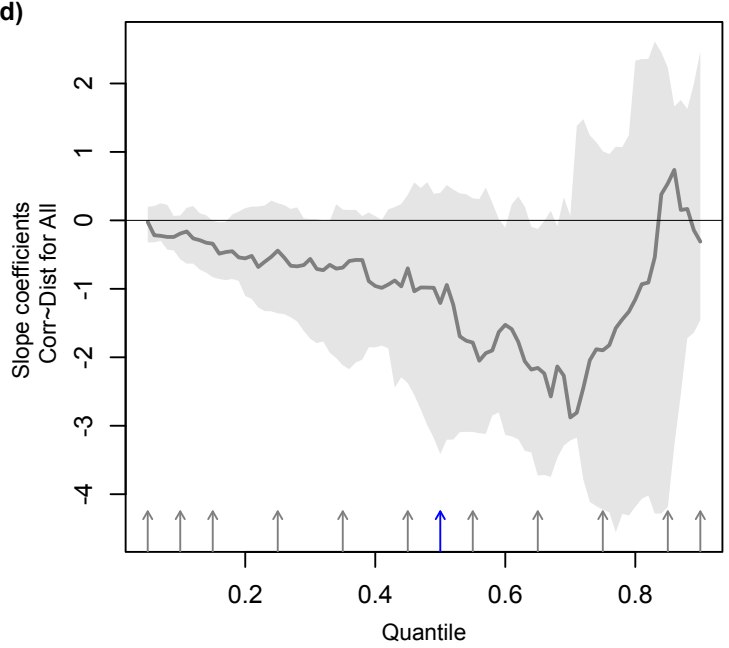
and D. (A, C) Scatterplots of LVM co-responses versus LVM residuals superimposed with the lines for $5^{\text {th }}, 10^{\text {th }}, 15^{\text {th }}, 25^{\text {th }}, 35^{\text {th }}, 45^{\text {th }}, 55^{\text {th }}, 65^{\text {th }}, 75^{\text {th }}, 85^{\text {th }}$ and $90^{\text {th }}$ quantile regression fits (grey lines), the median fit ( $50^{\text {th }}$ quantile; blue line), and the least squares estimate or mean fit (dashed red line). (B, D) Panels show the quantile slope estimates (dark grey line) and corresponding confidence intervals (light grey bands) of the response models across all quantiles (arrows, with the median shown in blue). Significant quantile slopes occur when confidence intervals do not overlap zero. 
397 The observed tendency for more closely-related Synechococcus to share similar realized niches

398 (Figure 2A) could be explained by shared preferences for either unmeasured abiotic factors or

399 biotic interactions. We used co-occurrence network analysis to explore the role of potential biotic

400 interactions. First, we confirmed that more closely related Synechococcus tend to co-occur across

401 samples. We observed a negative relationship between node co-occurrence and pairwise genetic

402 distance (Figure 3), indicating that genetically similar Synechococcus nodes are indeed more

403 likely to co-occur, and thus to have similar realized niches. This pattern was significant [linear

404 regression, $\mathrm{F}(1,488)=108.6, P<0.001$, adjusted $\left.\mathrm{R}^{2}=0.18\right]$.

405

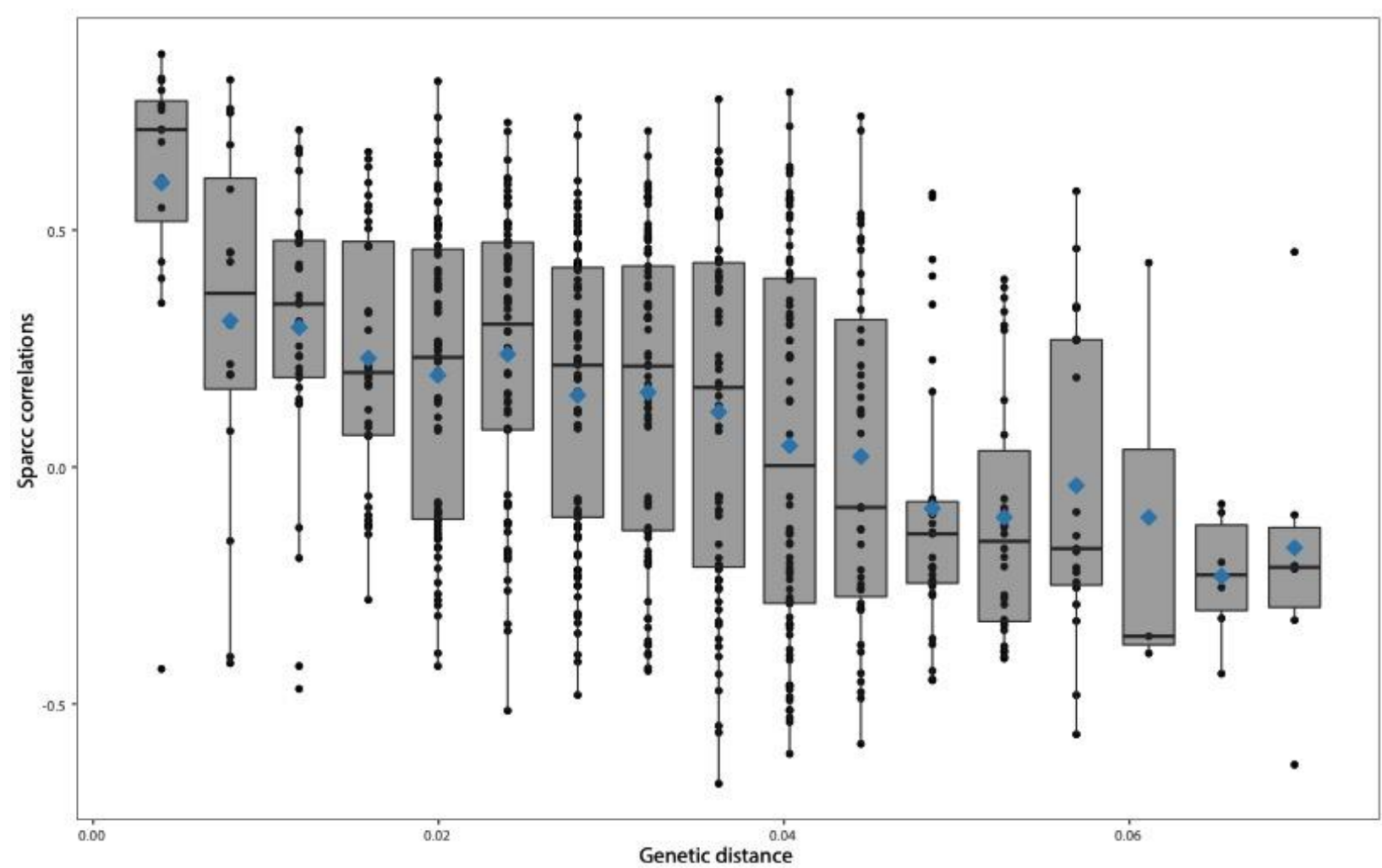

407 Figure 3. Negative relationship between pairwise SparCC correlation coefficients and 408 pairwise genetic distance between Synechococcus nodes. Only significant SparCC correlations $409(\mathrm{Q}<0.05)$ were included. Pairwise genetic distance was computed as percent nucleotide identity 
410 (p-distance). Blue diamonds represent the mean SparCC correlation for each distance. Boxplots

411 show the median (horizontal line), the 25th and 75 th percentile (enclosed in box) and $95 \%$

412 confidence intervals (whiskers).

414 If more closely-related Synechococcus tend to co-occur, it follows that they should also co-occur

415 with more similar surrounding microbial communities. The composition of the surrounding

416 microbial community could provide information about the nature of the realized niche, while also

417 suggesting possible biotic interactions. To confirm the expectation that more closely-related

418 Synechococcus should also share similar surrounding communities, we performed a pairwise

419 analysis to examine the relationship between Synechococcus genetic distance and $|\Delta r|$, a measure

420 of the similarity of Synechococcus co-occurrence with non-Synechococcus taxa (Methods). A

421 total of 373 non-Synechococcus taxa were analysed and the correlation between $|\Delta r|$ and genetic

422 distance was non-significant for 120 of them. The remaining 253 significant non-Synechococcus

423 have consistently positive relationships between co-occurrence and genetic distance, i.e. more

424 closely related Synechococcus nodes have a higher chance of co-occurring with similar non-

425 Synechococcus taxa (Figure 4). This result is robust to data structure, as determined by permuting

426 the associations between genetic distance and $|\Delta r|$ (Figure S13; Methods). 


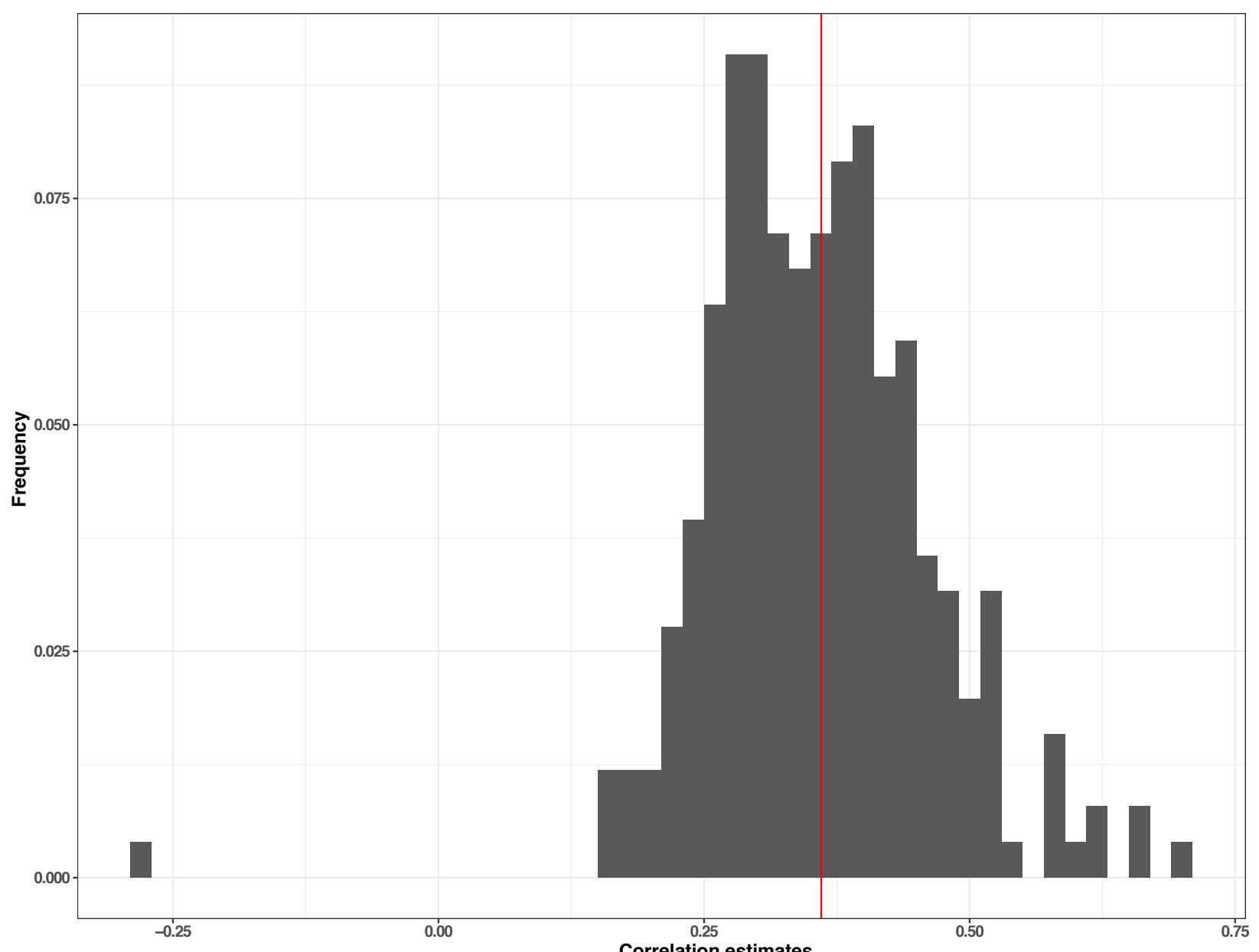

430 Figure 4. More closely related Synechococcus nodes co-occur with similar surrounding

431 community. The histogram shows the distribution of Spearman correlations between the genetic

432 distance of Synechococcus nodes and their association with similar surrounding communities.

433 Spearman correlations were calculated between the genetic distance of Synechococcus pairs and

434 the absolute difference of SparCC correlation (r) of a Synechococcus node with a specific taxon

$435 \mathrm{~T}$, such that $|\Delta \mathrm{r}|=\left|\operatorname{Corr}\left(\mathrm{X}_{1}, \mathrm{~T}\right)-\operatorname{Corr}\left(\mathrm{X}_{2}, \mathrm{~T}\right)\right|$ where Corr is defined here as the SparCC

436 correlation score of $\mathrm{X}_{\mathrm{i}}$ (Synechococcus node) and $\mathrm{T}$ (non-Synechococcus node). We then

437 estimated the correlation of $|\Delta \mathrm{r}|$ and the genetic distance between nodes $\mathrm{X}_{1}$ and $\mathrm{X}_{2}$. The mean

438 Spearman correlation estimate was 0.373 (red line) and $99.6 \%$ of correlations are positive.

440 Finally, we selected the non-Synechococcus taxa with the highest correlation scores between $|\Delta \mathrm{r}|$

441 and Synechococcus distance genetic. Five taxa had correlation estimates higher than 0.6, 
442 including three Proteobacteria, one Verrucomicrobia, and one Planctomycetes (Table S1).

443 Previous studies have shown that members of the Comamonadaceae family and Spirobacillales

444 order (members of Proteobacteria; Table S1) were associated with Synechococcus (Guedes et al.,

445 2018) or more broadly with algae (Fullbright et al., 2018). Moreover, the Gallionella genus was

446 previously observed colonizing filamentous algae (Mori et al., 2015).

448 Overall, these results show that the co-occurring surrounding community is, on average, more

449 similar for more closely related Synechococcus. Members of this surrounding community could

450 interact directly with Synechococcus, or share similarity in their abiotic niches.

452 Discussion

453 In this study, we investigated Synechococcus niche preferences at relatively fine (sub454 genus) taxonomic resolution using a $16 \mathrm{~S}$ sequencing approach previously applied to other 455 bacterial lineages (Koeppel and $\mathrm{Wu}, 2014$; Tromas et al., 2018). However, we acknowledge that 456 even at the finest possible taxonomic resolution, the $16 \mathrm{~S}$ gene may be too slow-evolving to be a 457 good marker for fast-evolving traits, such as toxin production (Berry et al., 2017) or 458 bacteriophage resistance (Martiny et al., 2015). We found that water temperature, reservoir depth, 459 and Nitrite concentration greatly affected the relative abundance of Synechococcus strains (Figure 460 S6, S7). However, we did not find significant linear or non-linear relationships between genetic 461 distance and preference for any of these abiotic niches. This was true when considering the whole 462 range of genetic distance ( $>93 \%$ identity) or a restricted range of more closely related strains $463(>97 \%)$, suggesting that these traits are phylogenetically unconserved (at least within our dataset 464 and at the resolution of the $16 \mathrm{~S}$ marker). Such a lack of conservation would be expected for a trait 465 such as Nitrite or Nitrate utilization in an organism capable of fixing nitrogen. Although some 
marine Synechococcus can fix nitrogen (Zehr et al., 2001), further investigation will be needed to

467 determine if this is also the case in our freshwater community.

In contrast, total phosphorus (TP) preference was more phylogenetically constrained, even

469 though it explained less variation in community structure than Nitrite (Figure S6, S7).

470 Phosphorus use may therefore evolve "clock-like" along the Synechococcus phylogeny, following

471 an approximately linear model in the range of 96-100\% nucleotide identity (Figure 1). This

472 contrasts with the observation in another cyanobacterium (Prochlorococcus) that organic

473 phosphate niches are fast-evolving and thus correlated with the phylogeny only near the tips of

474 the phylogenetic tree (Coleman \& Chisholm 2010; Martiny et al., 2015). Consistent with this

475 idea, we identified significant niche separation among very closely related ( $99 \%$ identical in

476 16S) Synechococcus, although the effect was driven by only three pairs of strains (Figure S12).

477 We also note that TP is a fairly crude measurement, encompassing both organic and inorganic

478 forms of phosphorus, and adaptation to these finer-grained niche dimensions may evolve

479 differently in Synechoccus. TP may not even be the driving selective pressure and may simply be

480 a marker of another important factor (e.g. runoff, overall biomass, etc.). Furnas Reservoir is

481 meso-eutrophic and phosphorus is probably not limiting (concentration varies from 8 to $53 \mu \mathrm{g} / \mathrm{L}$,

482 with a mean $\sim 25 \mu \mathrm{g} / \mathrm{L})$. This might explain why only 77 of the 780 Synechococcus strain pairs

483 showed a significant co-response to phosphorus (Figure 1), indicating that the effect is driven by

484 a small number of closely-related strains with similar co-responses, and cannot be generalized to

485 all Synechococcus. This result might also be explained if Synechococcus represents a 486 polyphyletic group (Coutinho et al., 2016), composed of different clades with different 487 phosphorus niches. However, in our sample, Synechococcus is monophyletic (Figure S3, S4), 488 excluding this explanation. Overall, our results demonstrate how abiotic factors can shape 489 community composition within a genus but measured abiotic niches generally do not evolve 
490 along the genus-level phylogeny.

491

492 Habitat filtering was originally defined to describe how abiotic factors select for genetically

493 similar (more closely related) organisms (Cadotte and Tucker 2017). Yet in practice, it is difficult

494 to disentangle the contributions of biotic and abiotic factors, and thus to satisfactorily define

495 habitat filtering (Kraft et al., 2015). In this study, we therefore estimated the influence of both

496 unmeasured abiotic variables and biotic factors, and found a negative association with genetic

497 distance (Figure 2). This suggests that more closely related Synechococcus tend to share similar

498 realized niches. As a result, we confirmed that genetically similar Synechococcus nodes tend to

499 co-occur, (Figure 3), as observed in studies using genomic (rather than 16S) similarity (Kamneva,

500 2017). This result could be unintuitive especially if Synechococcus nodes co-occur, but do not

501 share abiotic dimensions. For example, two taxa could be present in the same samples, but at very

502 different relative abundances. Therefore, they would co-occur but respond quantitatively to

503 different environmental variables. Overall, this result is consistent with habitat filtering, broadly

504 defined to include both biotic and abiotic factors.

506 Here we found that commonly-measured abiotic environmental factors (e.g. nutrients,

507 temperature) may be insufficient to capture relevant niche dimensions, and biotic co-occurrence

508 networks could be more informative about the evolution of niche preferences. In this study, we

509 measured abiotic factors such as temperature and nutrients, which are important in structuring

510 microbial communities, and yet these factors are largely insufficient to account for realized

511 niches or their distribution across the Synechococcus phylogeny. However, more closely-related

512 Synechococcus tend to co-occur with more similar surrounding microbial communities. As

513 previously suggested, the surrounding microbial community could provide hints as to the nature 
514 of a realized niche (Cohan and Koeppel, 2008; Tromas et al., 2018). For example, the presence of

515 certain taxa could indicate the importance of an unmeasured abiotic factor (e.g Acidophiles or

516 Alkaliphiles as indicators of $\mathrm{pH}$ ). In some cases, co-occurrence may indicate real biological

517 interactions (e.g. physical association, predation, cross-feeding, etc.). Some of the bacteria whose

518 co-occurrence with Synechococcus was strongly phylogenetically conserved have previously

519 been found in physical association with algae and cyanobacteria (Table S1; Mori et al. 2015;

520 Fullbright et al., 2018) and future work could experimentally test the nature of possible

521 interactions with Synechococcus strains. Alternatively, co-occurring microbes could simply share

522 similar unmeasured niches (including unmeasured abiotic or biotic factors such as phage or

523 protozoan grazers), and mining their genome sequences or metabolic capabilities could provide

524 insight into the nature of these niches. In this case, considering biotic factors allowed us to

525 uncover a signal of habitat filtering within Synechococcus that would have been obscured by only

526 considering commonly measured abiotic factors. 


\section{References}

529 Allewalt, J.P., Bateson, M.M., Revsbech, N.P., Slack, K., and Ward, D.M. (2006) Effect of Temperature and Light on Growth of and Photosynthesis by Synechococcus Isolates Typical of Those Predominating in the Octopus Spring Microbial Mat Community of Yellowstone National Park. Appl Environ Microbiol 72: 544-550.

Becker, S., Richl, P., and Ernst, A. (2007) Seasonal and habitat-related distribution pattern of Synechococcus genotypes in Lake Constance. FEMS Microbiol Ecol 62: 64-77.

Becraft, E.D., Cohan, F.M., Kühl, M., Jensen, S.I., and Ward, D.M. (2011) Fine-Scale Distribution Patterns of Synechococcus Ecological Diversity in Microbial Mats of Mushroom Spring, Yellowstone National Park. Appl Environ Microbiol 77: 7689-7697.

Berry, M. A., White, J. D., Davis, T. W., Jain, S., Johengen, T. H., Dick, G. J., et al. (2017). Are oligotypes meaningful ecological and phylogenetic units? A case study of Microcystis in freshwater lakes. Front Microbiol $8: 365$.

Breiman L, Friedman JH, Olshen RA, Stone CJ. (1984) Classification and Regression Trees. Wadsworth International Group, Belmont, CA, USA.

Burke, C., Steinberg, P., Rusch, D., Kjelleberg, S., and Thomas, T. (2011) Bacterial community assembly based on functional genes rather than species. Proc Natl Acad Sci USA 108: $14288-14293$.

Cade, B.S. and Noon, B.R. (2003) A Gentle Introduction to Quantile Regression for Ecologists. Frontiers in Ecology and the Environment 1: 412-420.

Cadotte, M.W. and Tucker, C.M. (2017) Should Environmental Filtering be Abandoned? Trends in Ecology \& Evolution 32: 429-437.

Callieri, C. (1996) Extinction coefficient of red, green and blue light and its influence on picocyanobacterial types in lakes at different trophic levels. Memorie dell'Istituto Italiano di Idrobiologia 54: 135-142.

Callieri, C., Caravati, E., Corno, G., and Bertoni, R. (2012) Picocyanobacterial community structure and space-time dynamics in the subalpine Lake Maggiore (N. Italy). Journal of Limnology 71: e9-e9.

Callieri, C., Lami, A., and Bertoni, R. (2011) Microcolony Formation by Single-Cell Synechococcus Strains as a Fast Response to UV Radiation. Appl Environ Microbiol 77: 7533-7540.

Cohan, F.M. and Koeppel, A.F. (2008) The origins of ecological diversity in prokaryotes. Curr Biol 18: R1024-1034.

Coleman, M. L., and Chisholm, S. W. (2010). Ecosystem-specific selection pressures revealed 
through comparative population genomics. Proc. Natl. Acad. Sci. U. S. A. $107: 18634$ 18639.

Coutinho, F., Tschoeke, D.A., Thompson, F., and Thompson, C. (2016) Comparative genomics of Synechococcus and proposal of the new genus Parasynechococcus. PeerJ 4: e1522.

De'ath G. (2007) mvpart: Multivariate partitioning. R package version 1.6-2.

Dhariwal, A., Chong, J., Habib, S., King, I.L., Agellon, L.B., and Xia, J. (2017) MicrobiomeAnalyst: a web-based tool for comprehensive statistical, visual and metaanalysis of microbiome data. Nucleic Acids Res 45: W180-W188.

Edgar, R.C. (2010) Search and clustering orders of magnitude faster than BLAST. Bioinformatics 26: $2460-2461$.

Eren, A.M., Morrison, H.G., Lescault, P.J., Reveillaud, J., Vineis, J.H., and Sogin, M.L. (2015) Minimum entropy decomposition: unsupervised oligotyping for sensitive partitioning of high-throughput marker gene sequences. ISME J 9: 968-979.

Faust, K., Sathirapongsasuti, J.F., Izard, J., Segata, N., Gevers, D., Raes, J., and Huttenhower, C. (2012) Microbial Co-occurrence Relationships in the Human Microbiome. PLOS Computational Biology 8: e1002606.

Fernandes, A.D., Reid, J.N., Macklaim, J.M., McMurrough, T.A., Edgell, D.R., and Gloor, G.B. (2014) Unifying the analysis of high-throughput sequencing datasets: characterizing RNA-seq, 16S rRNA gene sequencing and selective growth experiments by compositional data analysis. Microbiome 2: 15.

Ferris, M.J., Kühl, M., Wieland, A., and Ward, D.M. (2003) Cyanobacterial Ecotypes in Different Optical Microenvironments of a $68^{\circ} \mathrm{C}$ Hot Spring Mat Community Revealed by 16S-23S rRNA Internal Transcribed Spacer Region Variation. Appl Environ Microbiol 69: $2893-2898$.

Fierer, N. and Jackson, R.B. (2006) The diversity and biogeography of soil bacterial communities. Proc Natl Acad Sci USA 103: 626-631.

Friedman, J. and Alm, E.J. (2012) Inferring Correlation Networks from Genomic Survey Data. PLOS Computational Biology 8: e1002687.

Fulbright, S.P., Robbins-Pianka, A., Berg-Lyons, D., Knight, R., Reardon, K.F., Chisholm, S.T. (2018) Bacterial community changes in an industrial algae production system. Algal Res 31:147-156.

Guindon, S., Dufayard, J.-F., Lefort, V., Anisimova, M., Hordijk, W., and Gascuel, O. (2010) New algorithms and methods to estimate maximum-likelihood phylogenies: assessing the performance of PhyML 3.0. Syst Biol 59: 307-321. 
621

Green, J.L., Bohannan, B.J.M., and Whitaker, R.J. (2008) Microbial Biogeography: From Taxonomy to Traits. Science 320: 1039-1043.

Guedes, I.A., Rachid, C.T.C.C., Rangel, L.M., Silva, L.H.S., Bisch, P.M., Azevedo, S.M.F.O., and Pacheco, A.B.F. (2018) Close Link Between Harmful Cyanobacterial Dominance and Associated Bacterioplankton in a Tropical Eutrophic Reservoir. Front Microbiol 9: 424.

Horner-Devine, M.C. and Bohannan, B.J.M. (2006) Phylogenetic Clustering and Overdispersion in Bacterial Communities. Ecology 87: S100-S108.

Hui, F.K.C., Taskinen, S., Pledger, S., Foster, S.D., and Warton, D.I. (2015) Model-based approaches to unconstrained ordination. Methods in Ecology and Evolution 6: 399-411.

Hutchinson, G.E. (1957) Concluding Remarks. Cold Spring Harb Symp Quant Biol 22: 415-427.

Hutchinson, G.E. (1961) The Paradox of the Plankton. The American Naturalist 95: 137-145.

Kamneva, O.K. (2017) Genome composition and phylogeny of microbes predict their cooccurrence in the environment. PLOS Computational Biology 13: e1005366.

Katoh, K. and Standley, D.M. (2013) MAFFT multiple sequence alignment software version 7: improvements in performance and usability. Mol Biol Evol 30: 772-780.

Koeppel, A.F. and Wu, M. (2014) Species matter: the role of competition in the assembly of congeneric bacteria. ISME J 8: 531-540.

Koenker, R. (2015). Package 'quantreg'

Koenker, R., Machado, J.A.F. (1999). Goodness of fit and related inference processes for quantile regression. Journal of the American Statistical Association. 94, 1296-1310.

Kraft, N. J. B., Adler, P. B., Godoy, O., James, E. C., Fuller, S., and Levine, J. M. (2015). Community assembly, coexistence and the environmental filtering metaphor. Funct. Ecol. 29, 592-599.

Kumar, S., Stecher, G., and Tamura, K. (2016) MEGA7: Molecular Evolutionary Genetics Analysis Version 7.0 for Bigger Datasets. Mol Biol Evol 33: 1870-1874.

Kurmayer, R., Christiansen, G., and Chorus, I. (2003) The Abundance of Microcystin-Producing Genotypes Correlates Positively with Colony Size in Microcystis sp. and Determines Its Microcystin Net Production in Lake Wannsee. Appl Environ Microbiol 69: 787-795.

Larkin, A.A and Martiny, A.C. (2017) Microdiversity shapes the traits, niche space, and biogeography of microbial taxa. Environmental Microbiology Reports 9:55-70.

Letten, A.D., Keith, D.A., Tozer, M.G., and Hui, F.K.C. (2015) Fine-scale hydrological niche differentiation through the lens of multi-species co-occurrence models. Journal of 
Magurran, A.E. and Henderson, P.A. (2003) Explaining the excess of rare species in natural

Martiny, J.B.H., Jones, S.E., Lennon, J.T., and Martiny, A.C. (2015) Microbiomes in light of traits: A phylogenetic perspective. Science 350: aac9323.

Mori, J.F., Neu, T.R., Lu, S., Händel, M., Totsche, K.U., and Küsel, K. (2015) Iron encrustations on filamentous algae colonized by Gallionella-related bacteria in a metal-polluted freshwater stream. Biogeosciences 12: 5277-5289.

Needham, D. M., Fuhrman, J. A. (2016) Pronounced daily succession of phytoplankton, archaea and bacteria following a spring bloom. Nature Microbiology, 1: 16005.

Newton, R.J., Jones, S.E., Eiler, A., McMahon, K.D., and Bertilsson, S. (2011) A guide to the natural history of freshwater lake bacteria. Microbiol Mol Biol Rev 75: 14-49.

Nusch, E.A. (1980) Comparison of different methods for chlorophyll and phaeopigment determination. Arch. Hydrobiol. Beih. 14 : 14-36."

Pascual-García, A., Tamames, J., and Bastolla, U. (2014) Bacteria dialog with Santa Rosalia: Are aggregations of cosmopolitan bacteria mainly explained by habitat filtering or by ecological interactions? BMC Microbiol 14: 284.

Preheim, S.P., Perrotta, A.R., Martin-Platero, A.M., Gupta, A., and Alm, E.J. (2013) Distribution-based clustering: using ecology to refine the operational taxonomic unit. Appl Environ Microbiol 79: 6593-6603.

Price MN, Dehal PS, Arkin AP. (2009). FastTree: computing large minimum evolution trees with profiles instead of a distance matrix. Mol Biol Evol 26:1641-1650.

Rohwer, R.R., Hamilton, J.J., Newton, R.J., and McMahon, K.D. (2018) TaxAss: Leveraging a Custom Freshwater Database Achieves Fine-Scale Taxonomic Resolution. mSphere 3: e00327-18.

Röttjers, L. and Faust, K. (2018) From hairballs to hypotheses-biological insights from microbial networks. FEMS Microbiol Rev 42: 761-780.

Scanlan, D.J. (2003) Physiological diversity and niche adaptation in marine Synechococcus. In, Advances in Microbial Physiology. Academic Press, pp. 1-64.

Schloss, P.D., Westcott, S.L., Ryabin, T., Hall, J.R., Hartmann, M., Hollister, E.B., et al. (2009) Introducing mothur: Open-Source, Platform-Independent, Community-Supported Software for Describing and Comparing Microbial Communities. Appl Environ Microbiol 75: 7537-7541. 
715

Steunou, A.-S., Bhaya, D., Bateson, M.M., Melendrez, M.C., Ward, D.M., Brecht, E., et al. (2006) In situ analysis of nitrogen fixation and metabolic switching in unicellular thermophilic cyanobacteria inhabiting hot spring microbial mats. Proc Natl Acad Sci USA 103: 2398-2403.

Stockner, J., Callieri, C., and Cronberg, G. (2002) Picoplankton and Other Non-Bloom-Forming Cyanobacteria in Lakes. In, Whitton,B.A. and Potts,M. (eds), The Ecology of Cyanobacteria: Their Diversity in Time and Space. Dordrecht: Springer Netherlands, pp. 195-231.

Tromas, N., Taranu, Z.E., Martin, B.D., Willis, A., Fortin, N., Greer, C.W., and Shapiro, B.J. (2018) Niche Separation Increases With Genetic Distance Among Bloom-Forming Cyanobacteria. Front Microbiol $9: 438$

Tromas, N., Fortin, N., Bedrani, L., Terrat, Y., Cardoso, P., Bird, D., et al. (2017) Characterising and predicting cyanobacterial blooms in an 8-year amplicon sequencing time course. The ISME Journal 11: 1746-1763.

Vörös, L., Callieri, C., Balogh, K.V., and Bertoni, R. (1998) Freshwater picocyanobacteria along a trophic gradient and light quality range. Hydrobiologia 369: 117-125.

Warton, D.I., Blanchet, F.G., O'Hara, R.B., Ovaskainen, O., Taskinen, S., Walker, S.C., and Hui, F.K.C. (2015) So Many Variables: Joint Modeling in Community Ecology. Trends in Ecology \& Evolution 30: 766-779.

Webb, C.O., Ackerly, D.D., McPeek, M.A., and Donoghue, M.J. (2002) Phylogenies and Community Ecology. Annual Review of Ecology and Systematics 33: 475-505.

Zheng, Q., Wang, Y., Xie, R., Lang, A.S., Liu, Y., Lu, J., et al. (2018) Dynamics of Heterotrophic Bacterial Assemblages within Synechococcus Cultures. Appl Environ Microbiol 84: e01517-17.

\section{1}

\section{Conflict of Interest}

753 The authors declare no conflict of interest. 


\section{Acknowledgments}

756 NT is funded by a project from the European Union's Horizon 2020 research and innovation

757 program under the Marie Sklodowska-Curie grant agreement No 656647. BJS was supported by

758 a Canada Research Chair and NSERC Discovery Grant. Sampling and samples processing were

759 supported by a grant from Furnas Centrais Hidroelétrica S.A. (Brazil) to AG. JSMP and DAP

760 received a scholarship from CAPES (Coordenação de Aperfeiçoamento de Pessoal de Nível

761 Superior). A sabbatical visit of AG to the Université de Montréal AG was supported by a CAPES

762 Senior Fellowship.

763 

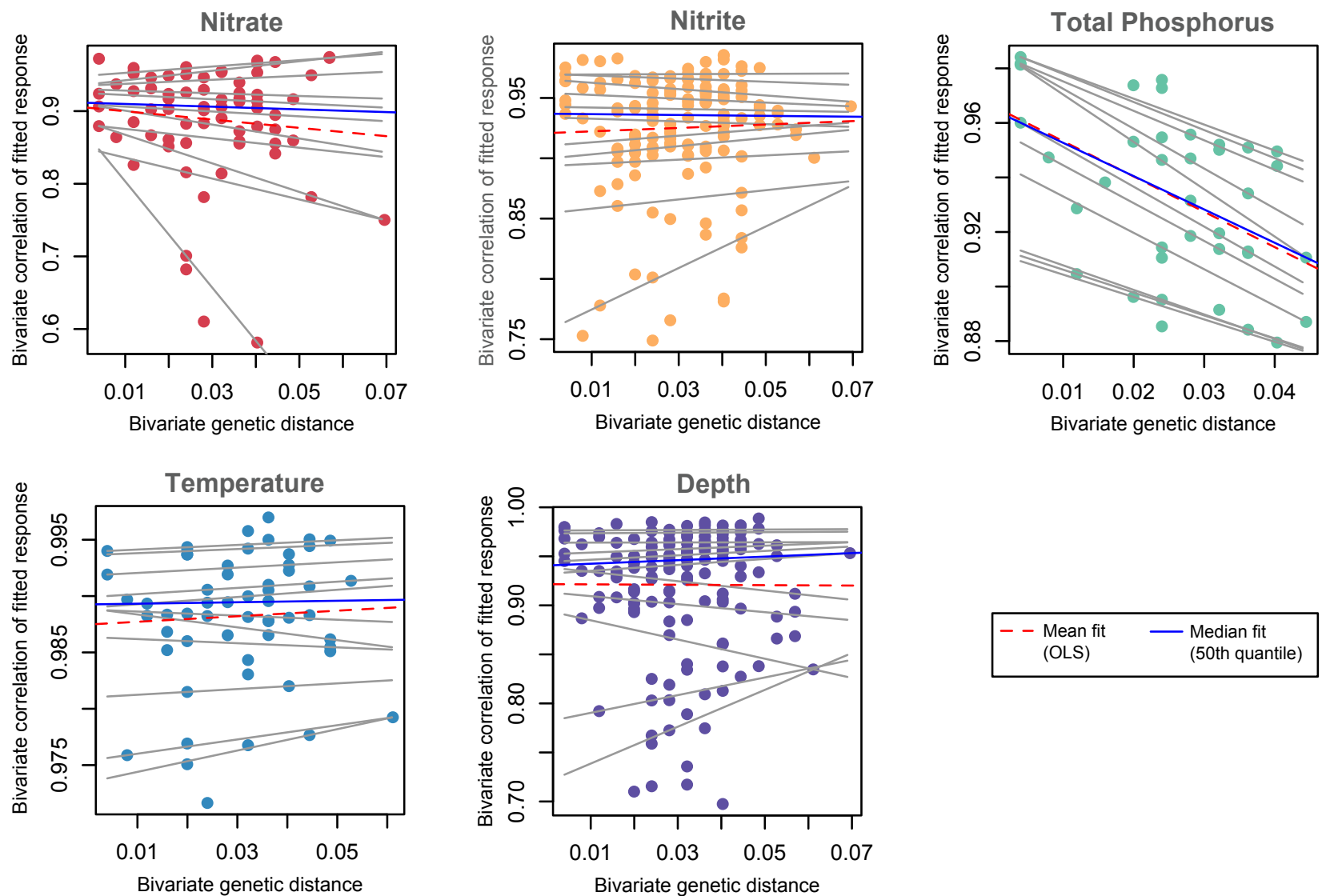

Bivariate genetic distance

Bivariate genetic distance 
(a)

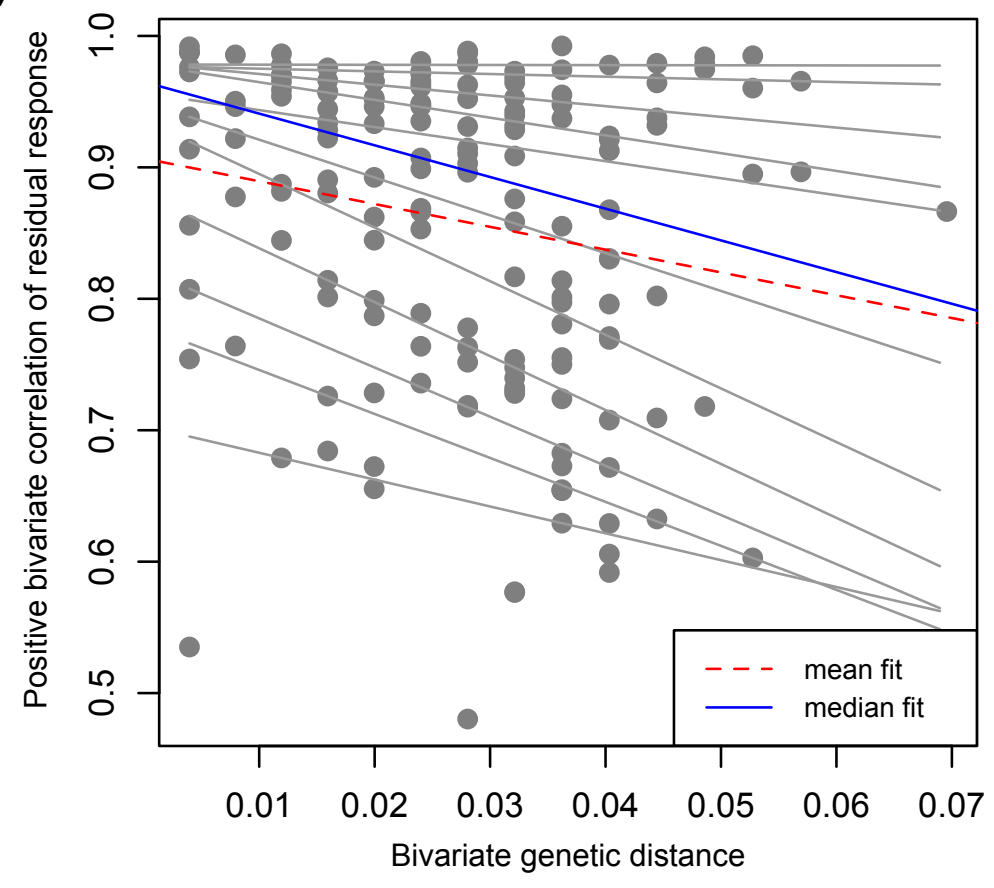

(b)

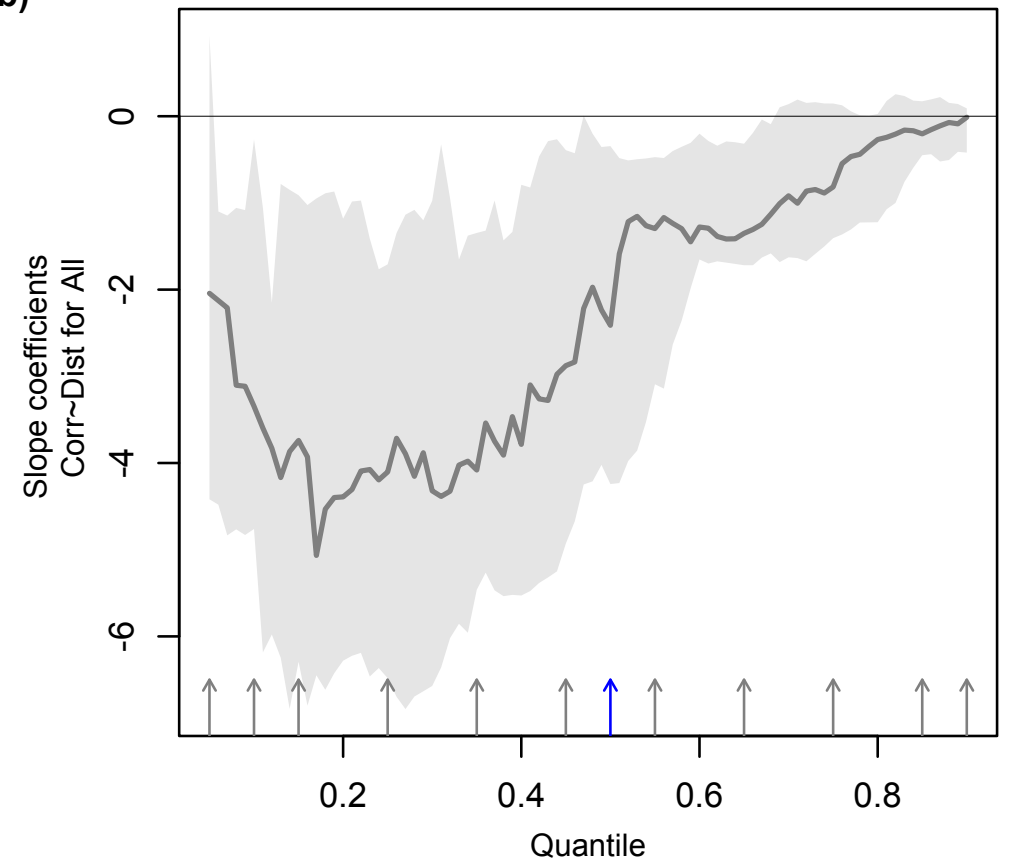

(c)

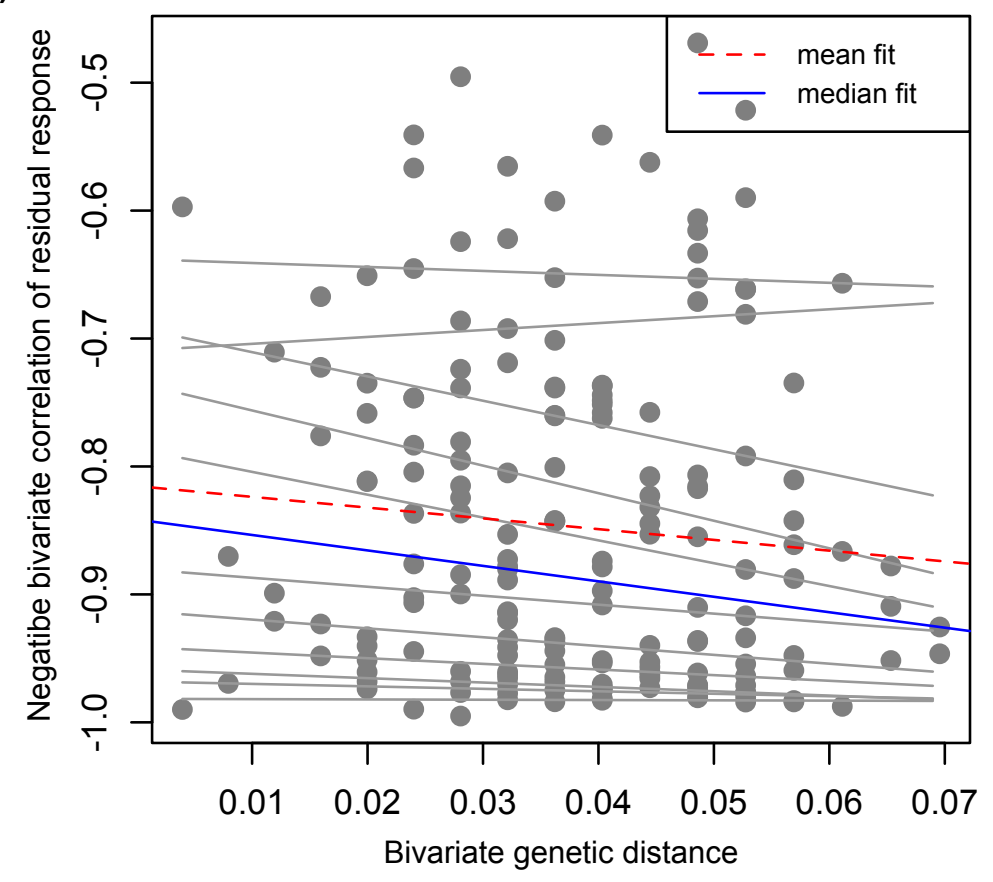

(d)

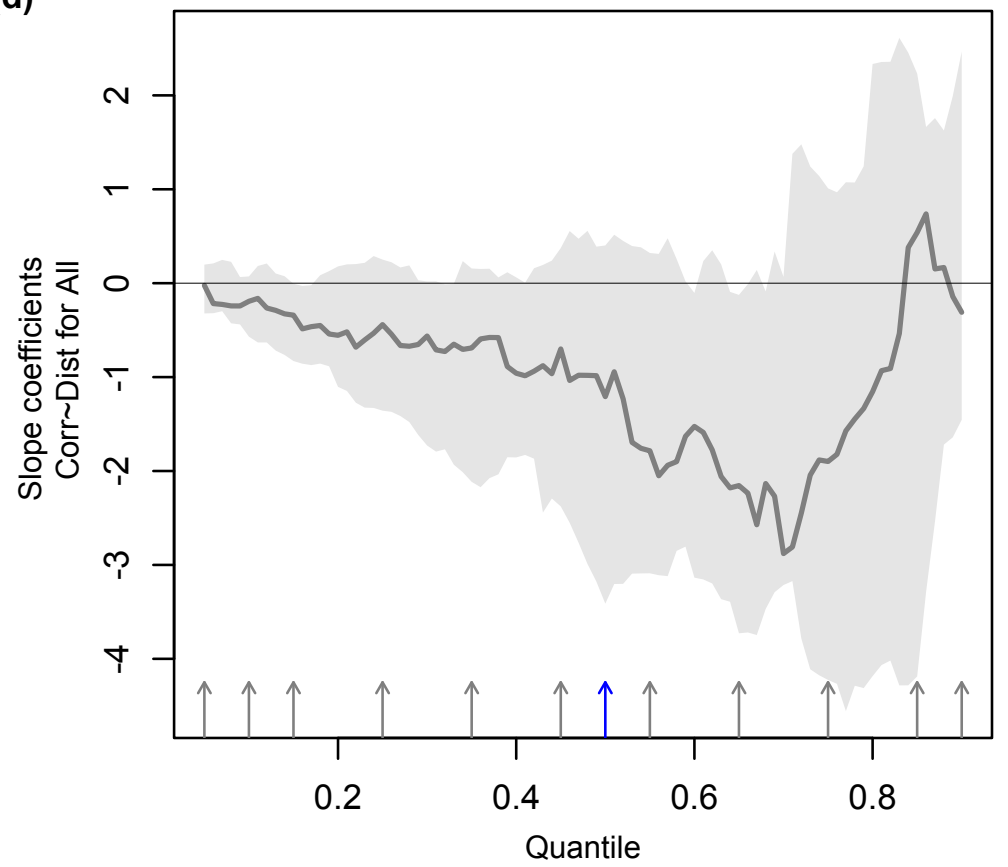




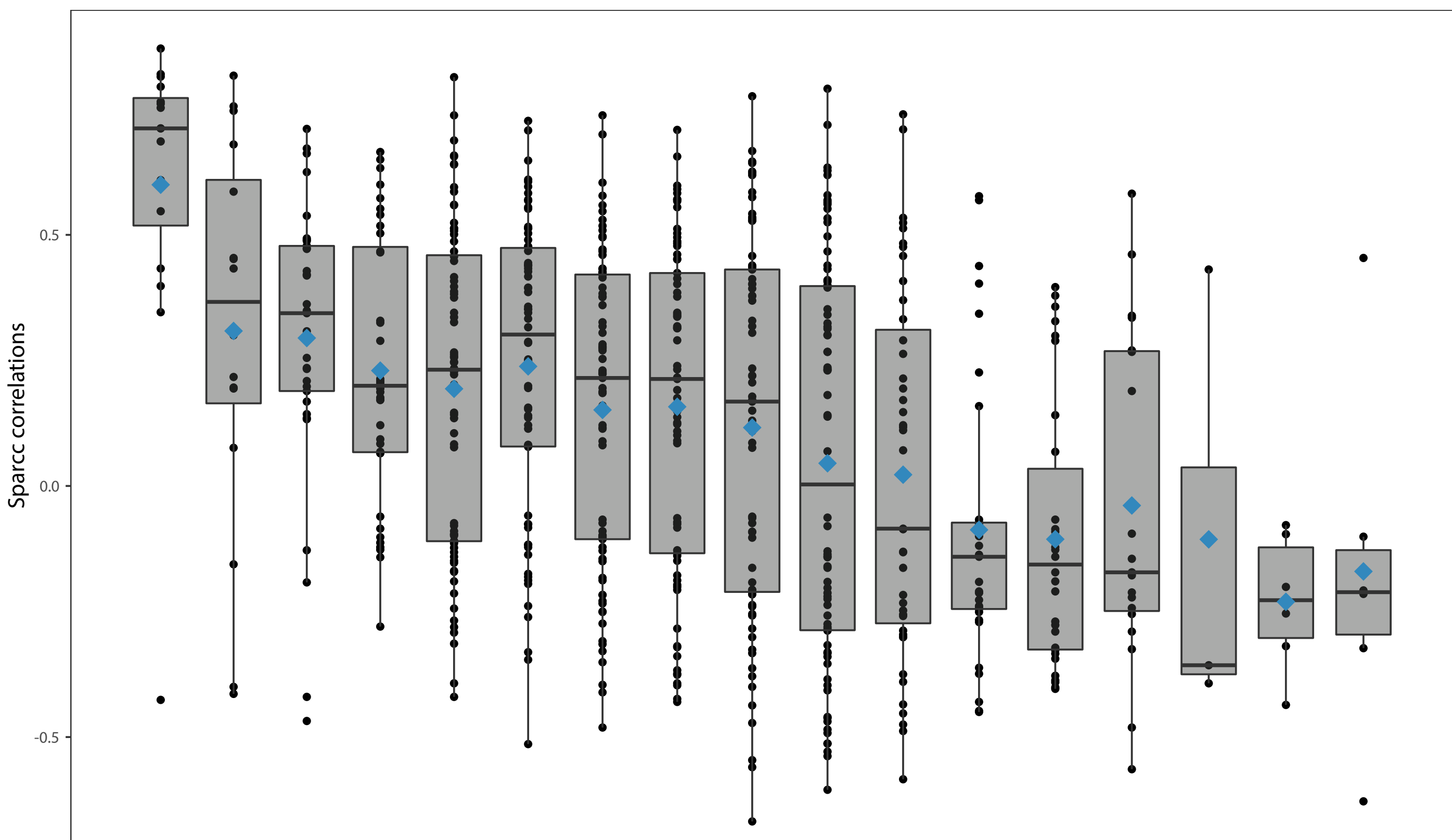


